\title{
The Extended Posterior Circumferential Decompression Technique in the Management of Tubercular Spondylitis with and without Paraplegia
}

\author{
Barani Rathinavelu, Justin Arockiaraj, Venkatesh Krishnan, Rohit Amritanand, Gabriel David Sundararaj
}

Department of Orthopaedics, Christian Medical College and Hospital, Vellore, India

Study Design: Retrospective clinical series.

Purpose: To study the clinical, functional and radiological results of patients with tuberculous spondylitis with and without paraplegia, treated surgically using the "Extended Posterior Circumferential Decompression (EPCD)" technique.

Overview of Literature: With the increasing possibility of addressing all three columns by a single approach, posterior and posterolateral approaches are gaining acceptance. A single exposure for cases with neurological deficit and kyphotic deformity requiring circumferential decompression, anterior column reconstruction and posterior instrumentation is helpful.

Methods: Forty-one patients with dorsal/dorsolumbar/lumbar tubercular spondylitis who were operated using the EPCD approach between 2006 to 2009 were included. Postoperatively, patients were started on nine-month anti-tuberculous treatment. They were serially followed up to thirty-six months and both clinical measures (including pain, neurological status and ambulatory status) and radiological measures (including kyphotic angle correction, loss of correction and healing status) were used for assessment.

Results: Disease-healing with bony fusion (interbody fusion) was seen in $97.5 \%$ of cases. Average deformity (kyphosis) correction was $54.6 \%$ in dorsal spine and $207.3 \%$ in lumbar spine. Corresponding loss of correction was 3.6 degrees in dorsal spine and 1.9 degrees in the lumbar spine. Neurological recovery in Frankel B and C paraplegia was $85.7 \%$ and $62.5 \%$, respectively.

Conclusions: The EPCD approach permits all the advantages of a single or dual session anterior and posterior surgery, with significant benefits in terms of decreased operative time, reduced hospital stay and better kyphotic angle correction.

Keywords: Extended posterior approach; Circumferential spinal canal decompression; Kyphosis correction; Interbody fusion; Neurological recovery

\section{Introduction}

Spinal Tuberculosis is one of the significant extra pulmonary forms of tuberculosis, with increased morbidity and mortality due to associated complications. Often, verte- bral destruction, cold abscess, epidural abscess, neurological deficits and kyphotic deformity of varying degrees, occurring in isolation or combination, warrant surgical management depending on the severity [1].

Traditionally, vertebral body problems related to tu-

Received Dec 15, 2013; Revised Jan 27, 2014; Accepted Jan 29, 2014

Corresponding author: Justin Arockiaraj

Department of Orthopaedics, Christian Medical College and Hospital, Vellore, Tamil Nadu, India. Pin 632004

Tel: +91-9443114380, Fax: +91416-2232054, E-mail: svjustin.arockiaraj@gmail.com 
mour, infection and trauma have been conventionally managed by the anterior approach [2]. With increasing awareness of the possibility for addressing all three columns by a single approach, posterior or posterolateral approach is gaining more acceptance. A variety of appropriate procedures such as laminectomy, transpedicular decompression, costotransversectomy and lateral extra cavitatory decompression have been dealt with through the posterior approach [3]. The extent of surgical exposure and exploration varies. Laminectomy deals with only the posterior column, whereas extra cavitatory approach deals mainly with the anterior column. A single technique to address all three columns adequately is very helpful in the presence of neurological deficit and kyphotic deformity, where circumferential decompression, anterior column reconstruction and posterior instrumentation are needed.

In this study, we describe a new posterior procedure termed the "Extended Posterior Circumferential Decompression (EPCD)" technique for 360 degrees dural decompression, anterior column debridement and reconstruction following posterior instrumentation, in the management of tubercular spondylitis with or without paraplegia. We discuss our results of treating forty-one cases by this approach, with the follow-up of up to three years.

\section{Materials and Methods}

This study was carried out as a retrospective outcome analysis of prospectively collected data, for a new approach in surgical management of tubercular spondylitis, with and without paraplegia.

From the records of tubercular spondylitis patients who were treated at the Christian Medical College (Vellore, India) from January 2006 till July 2009, details of patients with following inclusion criteria were considered for analysis. Patients having a histo-pathological or microbiological confirmed (confirmed with acid fast bacilli staining with Ziehl Neelsen staining method and culture growth in mycobacteria growth indicator tube method or growth in Lowenstein Jensen medium) diagnosis of Tubercular Spondylitis with varying severity of paraplegia; and significant vertebral body destruction, pain and deformity (kyphosis $>40$ degrees) were considered for the study. Patients less than thirteen years old, those with non contiguous multiple levels of vertebral affection, with irregular follow-up and history of defaulting anti-tubercular drug therapy were excluded from the study. During this period, all cases of dorsal, dorso-lumbar and lumbar tuberculous spondylitis cases operated for any indication were performed using this technique.

Severity of paraplegia was based on the functional impairment, as documented using Frankel scale [4]. Visual analog scale (VAS) scale was used to document back pain at admission and subsequently at follow-up. Kyphus angle (Konstam's) [5] was measured between the upper end plate of the above normal and inferior end plate of inferior normal vertebra, using Centricity 1.0 (RadiologyWeb, General Electric, Mount Prospect, Milwaukee, WI, USA).

Patients with one or more of the described inclusion criteria underwent surgical debridement, decompression and anterior column reconstruction with bone graft or mesh cage by the EPCD technique. All patients were given anti-tubercular drugs for nine months (two months of rifampicin+isoniazid+pyrazinamide +ethambutol [HRZE], followed by seven months of rifampicin+isoniazid [HR]) [6].

All patients were followed-up at three-month intervals till nine months, and six-month interval for the following year and thereafter yearly with clinical assessment and radiological evaluation. Changes in pain, neurological status and deformity correction were documented using VAS scale, Frankel Scale and kyphosis angle measurement, respectively. Healing of the lesion was confirmed by appearance of signs of fusion, i.e., sentinel sign [7], when a metal cage was used, or with appearance of continuing trabecular pattern of dense cortical bone across the disc space from the body above to the body below. CT scan was done for confirmation of fusion or otherwise in cases with difficulty, in assessing trabecular pattern in plain radiographs [8].

\section{Operative procedure}

Patients were positioned prone, and a midline skin incision long enough to expose two to three vertebral segments caudal and cephalad to the involved vertebra was made. Appropriate levels were exposed and instrumented using pedicle screws. Generally, two levels cephalad and two levels caudal were instrumented for pedicle screw fixation. For Hartshill rectangle and sub-laminar wire fixation, three levels cephalad and caudal were wired. Bilat- 
eral costo transversectomy of the involved vertebral level/ levels was done to gain access to the prevertebral space (Fig. 1). After temporary stabilization of one side using connecting rod (Fig. 2), laminectomy of the maximallydestroyed vertebra was done, and opposite-side pedicle was cut to gain access to the diseased vertebral body. The procedure was repeated with the temporary rod on the other side to achieve thorough circumferential decompression (Fig. 3). Care was taken to proceed slowly with caution during the correction/re-alignment to prevent 'stretch of the cord' by shortening of the spinal cord. In lumbar vertebral disease, facet joint, pars and transverse process excision (bilaterally) replaced the costo-transver-

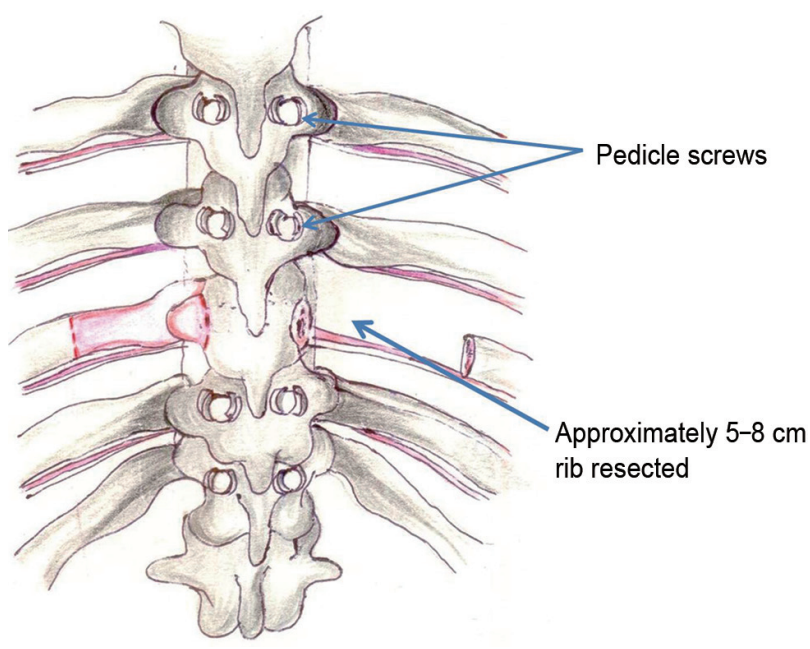

Fig. 1. Bilateral costo-transversectomy done to gain access to the prevertebral space. Approximately $5-8 \mathrm{~cm}$ of rib was resected.

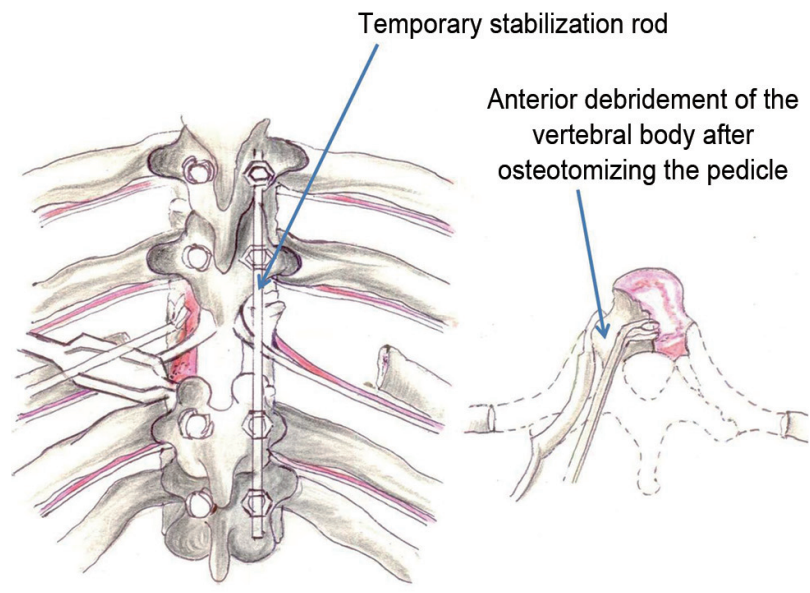

Fig. 2. Pedicle screw placement followed by temporary stabilization of one side with a rod. Decompression of the cord was done after laminectomy, and anterior debridement of the affected vertebra after osteotomizing the pedicle. sectomy in the dorsal and dorso-lumbar spine.

The anterior column defect was measured and reconstructed with metal (titanium) cages or tri cortical iliac crest graft (Fig. 4). Rod stabilization was completed and adequate anterior compression and correction achieved from the posterior aspect to get a stable construct. Intraoperative spinal cord monitoring with motor evoked potentials was used for patients with no or minimal neurological deficit.

The extent of correction/reduction is an intra-operative decision, guided by the neuro-monitoring device. Complete cessation of waveforms of motor evoked potentials in three successive recordings denotes insult to the cord and the amount of correction should be reduced from that point till the wave pattern reappears.

Duration of the procedure was documented as the time from skin incision to closure. Intra operative blood loss and replacements were carefully documented. In five cases with dorsal spine tuberculosis, sublaminar wires and Hartshill rectangle were used for stabilisation, as pedicle violation was expected even with the smallest available diameter screws. Rest of the thirty-six patients were posteriorly stabilized with only polyaxial-headed pedicle screws.

All patients, irrespective of their preoperative neurological status, were mobilized after drain removal (day 3-day 5). All patients were given parenteral antibiotics (3rd generation cephalosporin+gentamycin) till drain removal, following which anti-tubercular regimen was restarted. ATT was given for a total duration of nine months (2 HRZE+7 HR), irrespective of the duration of preoperative anti-tubercular therapy.

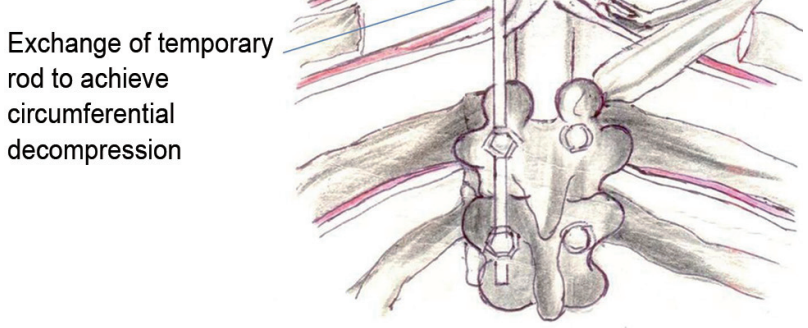

Fig. 3. Contralateral side was temporarily stabilised, and then the decompression was completed circumferentially from the other side. 


\section{Results}

There were 41 cases of tubercular spondylitis in the study period that satisfied the inclusion criteria. Mean age of the study group was 37.5 years, with a mean follow-up of 36 months (range, 18-54 months). Major indication for the procedure was paraplegia in 24 patients (59\%), and contiguous vertebral body destruction with deformity in 17 patients (41\%). Tubercular spondylitis affection was predominantly in dorsal vertebrae (53.7\%), followed by dorso-lumbar (29.3\%) and lumbar (17\%) vertebrae. Neurological deficits were documented more in dorsal segments (54\%), followed by dorsolumbar (29\%) and lumbar segments (17\%) (Table 1).

Mean postoperative VAS for back pain was 2 at discharge. Mean blood loss of the procedure was 1,191 mL (range, 250-3,000 mL). Total duration of surgery was 247 minutes (range, 180-360 minutes). Mean hospital stay was 16 days (range, 10-35 days). Anterior column was reconstructed with cage in 31 cases and tricortical iliac crest in 10 cases. Posterior column was stabilised using pedicle screws in 36 patients and Hartshill rectangle
Table 1. Site-infective spondylitis

\begin{tabular}{lcc} 
Location & $\begin{array}{c}\text { No. of } \\
\text { patients }\end{array}$ & $\begin{array}{c}\text { Neurological } \\
\text { deficit (\%) }\end{array}$ \\
\hline Thoracic & 22 & 54 \\
$\begin{array}{l}\text { Thoracolumbar } \\
\text { (T11/T12 disc to T12L1 disc) }\end{array}$ & 12 & 29 \\
$\begin{array}{l}\text { Lumbar } \\
\text { (L1/L2 disc to L4/L5 disc) }\end{array}$ & 7 & 17 \\
\hline
\end{tabular}

(Surgicraft, Redditch, UK) [9] and sublaminar wires in 5 patients.

Mean preoperative kyphus angle for the dorsal spine was 31.5 degrees (range, 5.6 to 97.7 degree), which changed to 14.2 degree (range, 1 to 38 degree) postoperatively. At final follow-up, mean kyphus angle was 17.8 degree (range, 5.4 to 14 degree), which accounted for a loss of correction by 3.6 degree (range, 0 to 13.1 degree).

Mean preoperative kyphus angle for the lumbar spine was 8.22 degree (range, -18.1 to 16 degree), which changed to -8.8 degree (range, -20.2 to -1.8 degree) postoperatively. At final follow-up, mean kyphus angle
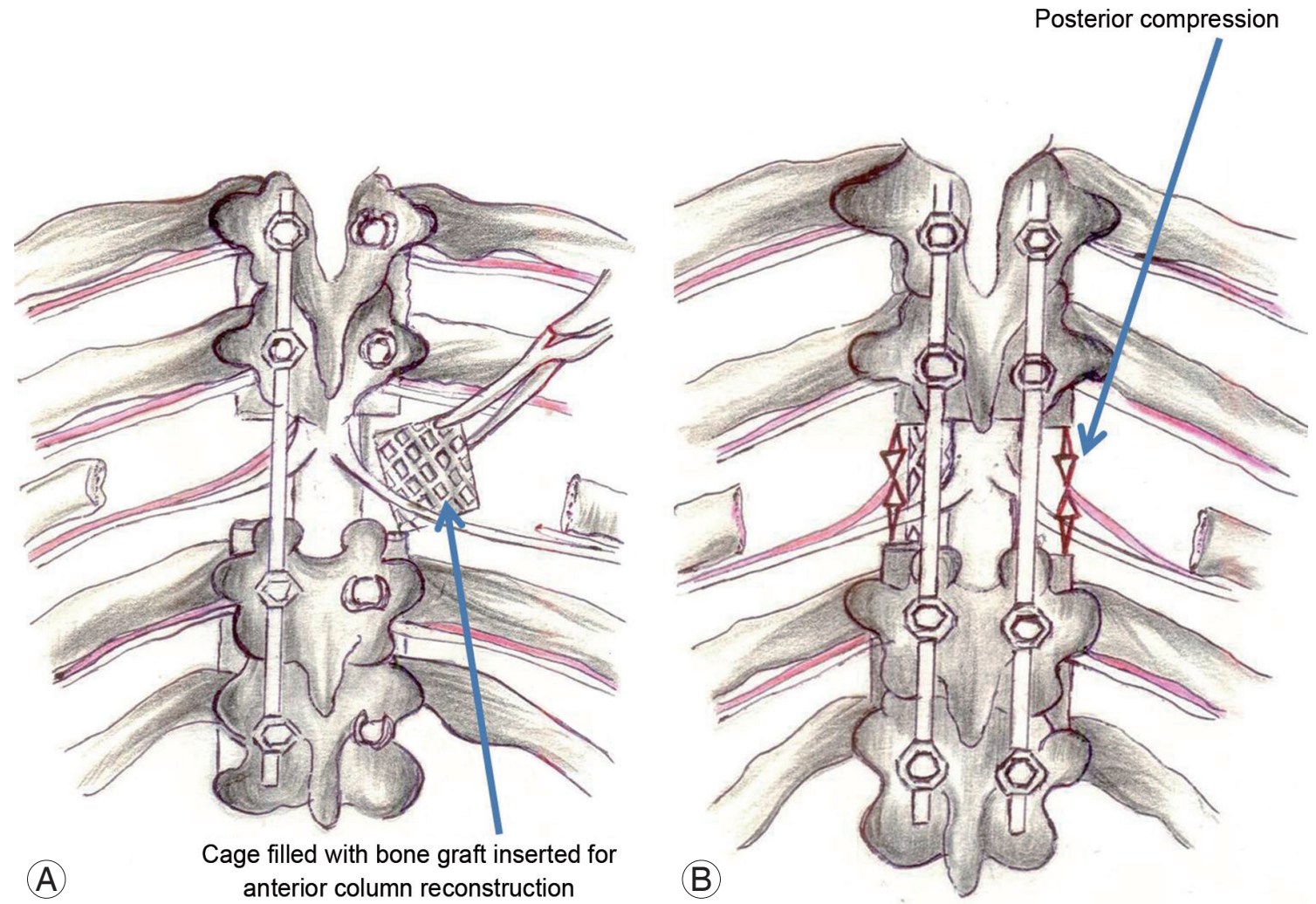

Fig. 4. (A, B) Anterior defect was measured and reconstructed with titanium cage filled with morselized autogenous cancellous graft and compressed posteriorly. 
Table 2. Deformity correction

\begin{tabular}{lccc} 
Level & Preoperative Kyphosis $\left({ }^{\circ}\right)$ & Postoperative kyphosis $\left({ }^{\circ}\right)$ & \% of correction \\
Thoracic & $31.5(5.6$ to 97.8$)$ & $14.3(1$ to 38$)$ & 54.6 \\
Thoracolumbar & $41.5(11.6$ to 71.3$)$ & $9.6(1$ to 18.3$)$ & 76.9 \\
\hline Lumbar & $8.2(-18.1$ to 16$)$ & $-8.8(-1.8$ to -20.2$)$ & 207.3 \\
\hline
\end{tabular}

Table 3. Neurological chart-Frankel grade

\begin{tabular}{lcc} 
Frankel & Preoperative & Final follow-up (36 mo) \\
A & 9 & 9 \\
B & 7 & 1 \\
C & 8 & 3 \\
D & 0 & 6 \\
E & 17 & 22 \\
\hline
\end{tabular}

was -6.3 degree (range, -19.4 to -1 degree), which accounted for a loss of correction by 1.9 degree (range, 0.5 to 3.9 degree). Correction of kyphotic angle was more significant in lumbar region, where the anterior column reconstruction was aimed to produce acceptable lordosis (Table 2).

Neurological assessment using Frankel scale showed significant change in $\mathrm{B}$ and $\mathrm{C}$ category of non-ambulatory paraplegics. Complete paraplegics (Frankel A) failed to show dramatic changes in motor deficit. There was transient worsening of motor deficit in one patient from Frankel C to B, which improved to Frankel D in two weeks. Two patients who worsened from Frankel B and $\mathrm{C}$ to Frankel A improved to Frankel $\mathrm{C}$ within three months (Table 3). At final follow-up, ambulatory status of paraplegics showed a change from $41.5 \%$ to $68.3 \%$ (statistically significant). Frankel B and C patients showed the maximal neurological recovery. Frankel A failed to show neurological improvement. The main reasons were: complete dense paraplegia (6 cases); duration of paraplegia averaging three months prior to surgery; past history of inadequate ATT (4 cases); and MRI demonstration of myelomalacia, cord edema and peridural fibrosis (all 9 cases).

Fusion was assessed by sentinel sign and dynamic radiographs. Evaluation by CT scan was done when radiographs were not confirmatory. CT scan evaluation was needed for 12 patients for confirmation of fusion; and 40 patients $(97.5 \%)$ showed fusion at final follow-up.

\section{Discussion}

Spinal tuberculosis continues to be the leading cause of spinal infection in developing countries. Even though there has been improvement in identification of the disease with improved laboratory services, radiological assessment, imaging techniques, surgical techniques and adequate facilities, a sizeable number of patients present late with complications of advanced disease. Management of such complications demands detailed assessment and proper surgical intervention, considering disease extent and co-morbidities.

The two most dreaded complications of spinal tuberculosis are paraplegia and kyphosis $[10,11]$. Often both complications co-exist, though they are mutually exclusive. Treatment strategies aim at decompression of spinal cord and restoration of normal alignment to create a microenvironment favourable to the cord's functional recovery and local disease healing. Kyphotic angle correction also facilitates restoration of global sagittal balance, having long-term implications on distant normal vertebrae. Hence deformity correction by anterior column reconstruction is equally important as spinal canal decompression to encourage neural recovery.

Abscess drainage and debridement with anterior column reconstruction and stabilisation, depending on the extent of destruction [12], form the corner stone in the presence of neurological deficit. Various approaches like isolated anterior [13], isolated posterior [14], double session anterior and posterior, single session anterior and posterior [12,15], single session single incision dual column approaching anterolateral [1] and posterolateral [16] approaches have been described to achieve this.

Isolated anterior approaches can achieve adequate spinal decompression, but the extent of deformity correction and maintenance with anterior implants is less [17]. Moreover they have higher rates of complications like injury to pleura, lung, abdominal viscera, paralytic ileus, injury to great vessels [18] and postoperative neurological 
Table 4. Comparative studies

\begin{tabular}{lcccc} 
Dual column procedures & $\begin{array}{c}\text { Moon et al. [23] } \\
\text { (staged, posterior } \\
\text { instrumentation+ } \\
\text { anterior approach) }\end{array}$ & $\begin{array}{c}\text { Laheri et al. [16] } \\
\text { (single stage- } \\
\text { posterolateral } \\
\text { decompression+anterior } \\
\text { column reconstruction) }\end{array}$ & $\begin{array}{c}\text { Jain et al. [1] } \\
\text { (single stage } \\
\text { anterolateral- } \\
\text { extra pleural } \\
\text { approach) }\end{array}$ & $\begin{array}{c}\text { Sundararaj et al. } \\
\text { (single stage-midline } \\
\text { "Extended Posterior" } \\
\text { approach)- } \\
\text { present study }\end{array}$ \\
\hline No. of cases & 33 & 28 & 38 & 41 \\
\hline Mean preoperative Kyphus angle $\left(^{\circ}\right)$ & 37 & 63.5 & 49.08 & 29.9 \\
\hline Mean Postoperative Kyphus angle $\left(^{\circ}\right)$ & 15 & 23.5 & 22.5 & 1.5 \\
\hline Mean loss of correction $\left(^{\circ}\right)$ & 3 & 3.2 & 51 & 2.7 \\
\hline Final follow up correction angle $(\%)$ & 51.3 & 58 & 61.5 \\
\hline
\end{tabular}

deficit of varying intensity [19]. Laminectomy as an isolated procedure for spinal decompression is contraindicated in tuberculosis, because of the inherent kyphosing nature of the disease which may contribute to increasing deformity, persistent back pain and delayed neurological worsening [20].

When deformity due to destruction and neurological deficit co-exist, a circumferential procedure often provides a comprehensive solution. An approach to remove devitalised diseased tissue and reconstruct the anterior column with adequate spinal decompression needs to be chosen. This should be guided by the general condition and existing co-morbidity in the patient. Anterior and posterior approaches either in single stage or two stages have been attempted by surgeons with variable success rates [12,15,21-24]. Morbidity of anterior procedures, namely excessive blood loss, delayed recovery and increased operative time may be considered as shortcomings of such dual approaches. Single session, single posterior midline incision, dual column procedure involving decompression, deformity correction and reconstruction in cases of paraplegia have possible advantage. Avoiding anterior approach avoids ligation of segmental vessels; and circumferential decompression leads to early recovery of compressed neural elements.

Single stage postero lateral and anterolateral approaches have the inherent potential of violation of thoracic cavity and injury to pleura and lungs which can delay recovery. Such complications can add to the morbidity associated with paraplegia. Deformity correction using such approaches in tubercular spondylitis is lower, compared to $61.5 \%$ corrected in the present technique (Table 4).

In this study, an isolated single stage posterior approach was performed to decompress the dural tube and recon- struct the anterior column, thereby addressing neurological deficit and kyphotic deformity.

Lateral extra cavitatory approach (postero lateral) [16] is being widely followed, where there is costotransversectomy, followed by anterior debridement and reconstruction. In this approach, prevertebral and epidural abscess is drained without laminectomy, which preserves the posterior tension band. Extent of deformity correction (51.3\%) preserving the posterior column is less compared to our technique, where posterior shortening has resulted in better deformity correction and global spinal alignment. Extra cavitatory approach involves lesser cord handling and reduces the chances of iatrogenic neurological deterioration, but the thoroughness of decompression is less compared to our technique, which creates wider posterior and lateral window by laminectomy and bilateral costotransversectomy. Wider space and exposure available reduces the need for intra-operative cord retraction and possible neurological worsening following surgery.

Antero-lateral extrapleural approach described by Jain et al. [1] involves anterior column reconstruction along with posterior Hartshill stabilisation in the lateral position. Although the results of deformity correction (51\%) and neurological improvement (89\%) are promising, this procedure can be technically demanding. The anterior column is approached after resecting three ribs using a T-shaped incision, which is likely to leave an unacceptable scar with a palpable bony defect on one side of rib excision.

Comparing our EPCD technique to other single incision single stage approaches $[1,16]$, our technique provides better deformity correction of $61.5 \%$ (Table 4 ). Both anterior column reconstruction and posterior column shortening contribute to this impressive correction. De- 
Table 5. Immediate postoperative complications

\begin{tabular}{lc} 
Value & Number (\%) \\
\hline Transient neurological deficit (C-B-E) & $1(2)$ \\
\hline Abdominal pain & $2(5)$ \\
\hline Transfusion reaction & $1(2)$ \\
\hline Excessive bleeding & $3(7)$ \\
\hline Dyselectrolytemia & $1(2)$ \\
Acute respiratory distress & $1(2)$ \\
\hline Pleural injury-ICD insertion & $1(2)$ \\
\hline Persistent intercostal neuralgia & $2(5)$ \\
\hline
\end{tabular}

ICD, inter-costal drain. formity correction was maximum in lumbar region where lordosis was recreated, followed by thoracolumbar and thoracic region (Table 2). Neurological improvement was noted in Frankel B and C paraplegics only. Nine Frankel A paraplegics failed to show neurological improvement, due to the presence of poor prognostic factors described earlier. At final follow-up, eleven (27\%) of non ambulant paraplegics became ambulant (Frankel A, B, C).

Average blood loss and operative time of our technique are comparable to anterolateral approach [1], but more than posterolateral approach [16]. Other complications like postoperative transient neurological worsening (2\%), transfusion reactions (2\%) and pleural injury which needed intercostal drain insertion (2\%) were monitored in intensive care units (Table 5). Mean duration of hos-
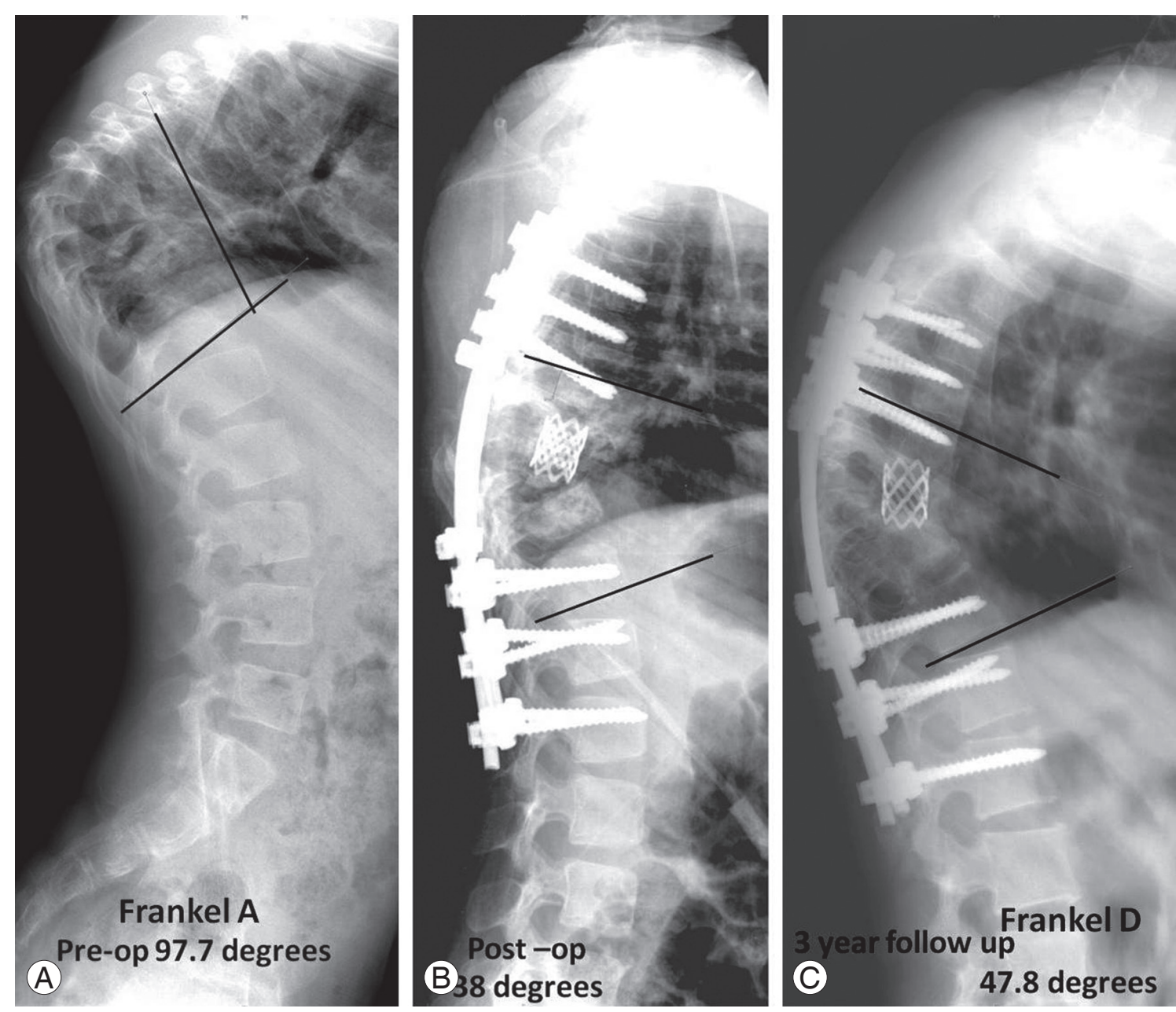

Fig. 5. (A-C) A 12-year-old boy, with destruction of D7, 8, 9 vertebra, was treated with first-line anti-tuberculosis drugs elsewhere for four months. He presented to us with progression of the disease, paraplegia Frankel A and kyphosis of 97.7 degrees. He was treated by extended posterior circumferential decompression. The culture was proven multidrug resistant tuberculosis; and the patient was treated with second line anti-tuberculosis drugs treatment for two years. At follow-up, his neurology recovered to Frankel D. Plain radiograph shows healed lesion with interbody fusion, kyphus angle of 47.8 degree kyphosis. The patient is presently pain-free and in near full-functional status. Pre-op, preoperative; Post-op, postoperative. 
pitalisation was sixteen days, which included postoperative rehabilitative care after suture removal at day ten of surgery.

Fusion assessed by appearance of sentinel sign (taken as a marker of local disease healing) was evident in $98 \%$ of cases. This radiological finding was correlated with haematological markers like erythrocyte sedimentation rate and C-reactive protein to confirm healing of disease.

An illustrative case treated by the above described technique along with the follow up xrays have been shown (Fig. 5).

\section{Conclusions}

Extended posterior technique permits circumferential decompression in tubercular spondylodiscitis. It achieves all the objectives of surgical management, i.e., decompression, debridement, deformity correction, spinal column stabilization and maintenance of suitable environment for neurological recovery and fusion comparable to anterior and posterior surgery (single or double session).

This novel approach also offers the advantage of reduced operative blood loss, shorter hospital stay and lower operative morbidity from avoidance of a separate anterior exposure. Deformity correction and its maintenance achieved by this single session, single incision technique and operative time are comparable to anterior and posterior (single/double session) surgeries.

\section{Conflict of Interest}

No potential conflict of interest relevant to this article was reported.

\section{Acknowledgments}

We would like to sincerely thank Dr. Christina Marie Joseph, Intern, Department of Orthopaedics, Christian Medical College and Hospital, Vellore for her wonderful art work in the illustration pictures to depict the various steps of the surgical technique described.

\section{References}

1. Jain AK, Dhammi IK, Prashad B, Sinha S, Mishra P. Simultaneous anterior decompression and posterior instrumentation of the tuberculous spine using an anterolateral extrapleural approach. J Bone Joint Surg Br 2008;90:1477-81.

2. Mehta JS, Bhojraj SY. Tuberculosis of the thoracic spine. A classification based on the selection of surgical strategies. J Bone Joint Surg Br 2001;83:859-63.

3. Zaveri G. The role of posterior surgery in spinal tuberculosis. ArgoSpine News J 2011;23:112-9.

4. Frankel HL, Hancock DO, Hyslop G, et al. The value of postural reduction in the initial management of closed injuries of the spine with paraplegia and tetraplegia. I. Paraplegia 1969;7:179-92.

5. Konstam PG, Blesovsky A. The ambulant treatment of spinal tuberculosis. Br J Surg 1962;50:26-38.

6. Upadhyay SS, Saji MJ, Yau AC. Duration of antituberculosis chemotherapy in conjunction with radical surgery in the management of spinal tuberculosis. Spine (Phila Pa 1976) 1996;21:1898-903.

7. McAfee PC. Interbody fusion cages in reconstructive operations on the spine. J Bone Joint Surg Am 1999;81:859-80.

8. Goldstein C, Drew B. When is a spine fused? Injury 2011;42:306-13.

9. Dove J. Internal fixation of the lumbar spine. The Hartshill rectangle. Clin Orthop Relat Res 1986;(203):135-40.

10. Rajasekaran $\mathrm{S}$. The problem of deformity in spinal tuberculosis. Clin Orthop Relat Res 2002;(398):8592.

11. Jain AK. Tuberculosis of the spine: a fresh look at an old disease. J Bone Joint Surg Br 2010;92:905-13.

12. Zhang HQ, Guo CF, Xiao XG, Long WR, Deng ZS, Chen J. One-stage surgical management for multilevel tuberculous spondylitis of the upper thoracic region by anterior decompression, strut autografting, posterior instrumentation, and fusion. J Spinal Disord Tech 2007;20:263-7.

13. Li M, Du J, Meng H, Wang Z, Luo Z. One-stage surgical management for thoracic tuberculosis by anterior debridement, decompression and autogenous rib grafts, and instrumentation. Spine J 2011;11:726-33.

14. Li C, He D, Hu W, Yang X, Ni Y, Li M. Modified single-stage transpedicular decompression, debridement, and posterior instrumentation in treatment of thoracic tuberculosis. Zhongguo Xiu Fu Chong Jian Wai Ke Za Zhi 2011;25:1294-7.

15. Sundararaj GD, Behera S, Ravi V, Venkatesh K, Che- 
rian VM, Lee V. Role of posterior stabilisation in the management of tuberculosis of the dorsal and lumbar spine. J Bone Joint Surg Br 2003;85:100-6.

16. Laheri VJ, Badhe NP, Dewnany GT. Single stage decompression, anterior interbody fusion and posterior instrumentation for tuberculous kyphosis of the dorso-lumbar spine. Spinal Cord 2001;39:429-36.

17. Ma YZ, Cui X, Li HW, Chen X, Cai XJ, Bai YB. Outcomes of anterior and posterior instrumentation under different surgical procedures for treating thoracic and lumbar spinal tuberculosis in adults. Int Orthop 2012;36:299-305.

18. Zahradnik V, Lubelski D, Abdullah KG, Kelso R, Mroz T, Kashyap VS. Vascular injuries during anterior exposure of the thoracolumbar spine. Ann Vasc Surg 2013;27:306-13.

19. Czerwein JK Jr, Thakur N, Migliori SJ, Lucas P, Palumbo M. Complications of anterior lumbar surgery. J Am Acad Orthop Surg 2011;19:251-8.

20. Rand C, Smith MA. Anterior spinal tuberculosis: paraplegia following laminectomy. Ann R Coll Surg Engl 1989;71:105-9.

21. Chen WJ, Wu CC, Jung CH, Chen LH, Niu CC, Lai PL. Combined anterior and posterior surgeries in the treatment of spinal tuberculous spondylitis. Clin Orthop Relat Res 2002;(398):50-9.

22. Klockner C, Valencia R. Sagittal alignment after anterior debridement and fusion with or without additional posterior instrumentation in the treatment of pyogenic and tuberculous spondylodiscitis. Spine (Phila Pa 1976) 2003;28:1036-42.

23. Moon MS, Woo YK, Lee KS, Ha KY, Kim SS, Sun $\mathrm{DH}$. Posterior instrumentation and anterior interbody fusion for tuberculous kyphosis of dorsal and lumbar spines. Spine (Phila Pa 1976) 1995;20:1910-6.

24. Talu U, Gogus A, Ozturk C, Hamzaoglu A, Domanic $\mathrm{U}$. The role of posterior instrumentation and fusion after anterior radical debridement and fusion in the surgical treatment of spinal tuberculosis: experience of 127 cases. J Spinal Disord Tech 2006;19:554-9. 